\title{
Level Shifts in Volatility and the Implied-Realized Volatility Relation
}

Bent J esper Christensen and Paolo Santucci de Magistris

\section{School of Economics and Management}

Aarhus University

Bartholins Allé 10, Building 1322, DK-8000 Aarhus C Denmark 


\title{
Level Shifts in Volatility and the Implied-Realized Volatility
}

\section{Relation*}

\author{
Bent Jesper Christensen ${ }^{\dagger}$ \\ Aarhus University and CREATES
}

Paolo Santucci de Magistris ${ }^{\ddagger}$

University of Pavia

September 10, 2010

\begin{abstract}
We propose a simple model in which realized stock market return volatility and implied volatility backed out of option prices are subject to common level shifts corresponding to movements between bull and bear markets. The model is estimated using the Kalman filter in a generalization to the multivariate case of the univariate level shift technique by $\mathrm{Lu}$ and Perron (2008). An application to the S\&P500 index and a simulation experiment show that the recently documented empirical properties of strong persistence in volatility and forecastability of future realized volatility from current implied volatility, which have been interpreted as long memory (or fractional integration) in volatility and fractional cointegration between implied and realized volatility, are accounted for by occasional common level shifts.
\end{abstract}

Keywords: Common level shifts, fractional cointegration, fractional VECM, implied volatility, long memory, options, realized volatility

JEL Classification: C32, G13, G14, G17

\footnotetext{
*We are grateful to CREATES (funded by the Danish National Research Foundation), D-CAF, and the Danish Social Science Research Council for research support. We thank Christian Bach for providing us with the data.

${ }^{\dagger}$ Correspondence: Bent Jesper Christensen, School of Economics and Management, Aarhus University, Bartholins Alle 10, 1322 University Park, DK-8000 Aarhus C, Denmark; phone: +45 89421 547; email address: bjchristensen@creates.au.dk

${ }^{\ddagger}$ Dipartimento di Economia Politica e Metodi Quantitativi, University of Pavia, Italy, email address: paolo.santuccidemagi01@ateneopv.it
} 


\section{Introduction}

The volatility of asset returns is among the most fundamental variables for asset pricing, derivative pricing, hedging, and risk management. Since future returns are valued and hedged, the forecasting of volatility of future returns from variables in the current information set is of particular importance. Given that observed prices of financial options are in the information set and reflect expectations of option market participants, implied volatility backed out of option prices should be informative about future volatility. Recent literature documents strong persistence in stock market volatility, whether measured as realized volatility from high-frequency returns within consecutive time intervals or as implied volatility from option prices at the beginning of each interval. Furthermore, the resulting realized and implied volatility series move together, so that implied volatility indeed predicts a considerable portion of the subsequently realized volatility. These findings have been interpreted as long memory or fractional integration in each of the two volatility series, and fractional cointegration between the two. The issue arises where such long memory should stem from, with current changes in volatility apparently depending on how volatility changed many periods back. In this paper, we propose a simple model in which realized and implied volatility are subject to occasional common level shifts, as could be generated, e.g., by changing bull and bear markets, movements between booms and busts, the recurrent onsets of financial crises, etc. This provides a plausible economic explanation of the received evidence.

The construction of a time series of realized volatility measures by basing volatility for each period, say, month, on returns measured at higher frequency during the course of the month follows Merton (1980), French et al. (1987) and Schwert (1989). The close relation between the monthly series of realized volatility and implied volatility backed out of stock index options at the beginning of the month is documented by Christensen and Prabhala (1998). Thus, implied volatility forecasts subsequent realized volatility, subsuming the information content of past realized volatility. Recent advances in data access has allowed basing realized measures on returns of higher frequency than the earlier daily returns, say, 5-minute returns, or transaction data. This allows very precise measurement of realized volatility simply as the sum of squared returns over the period, instead of the earlier sample standard deviation calculations, since the former converges to the integrated local volatility over the period in a general diffusion setting as sampling frequency is increased, see Barndorff-Nielsen and Shephard (2002). Using 
this high-frequency data technique for measurement, Andersen et al. (2001b) show that the resulting realized stock market volatility series is strongly persistent and is well described is a long memory or fractionally integrated process, with order of fractional integration in the vicinity of .4, i.e., more persistence than a stationary ARMA-type process, but less than a unit root process. Christensen and Nielsen (2006) show that the corresponding implied volatility series is similarly well approximated by a fractionally integrated process, and that the relation between implied and realized volatility may be described as one of fractional cointegration, i.e., the two long memory series move together, and a linear combination is serially uncorrelated, consistent with option prices incorporating all available information relevant for future volatility. This is shown using asymptotic theory for fractional cointegration developed for the purpose, and Bandi and Perron (2006) confirm the results using subsampling. Most recently, Busch et al. (2010) show that implied volatility backed out of a standard Black and Scholes (1973) style option pricing formula continues to forecast volatility better than past realized measures, even when the latter are adjusted for jumps in asset prices. Thus, the evidence is strong that volatility is persistent, and so should be forecastable, and that, indeed, the option-implied forecast is particularly important.

It is somewhat surprising that volatility changes in the distant past should matter so greatly for current volatility changes, as implied by a long memory process for volatility. If, instead, the empirical findings are the results of occasional level shifts in volatility, then the fractional integration characterization of each of the two volatility series should be replaced, and the fractional cointegration relation between implied and realized volatility should be reconsidered, as well. Thus, we introduce a model in which realized and implied volatility are linked by an underlying common level shift process. Our model extends the idea of co-breaking, see Hendry and Massmann (2007), to the fractional cointegration case, see also Morana (2002, 2007), showing that the results from the fractional cointegration analysis may be accounted for by common level shifts. Our common level shift model generalizes the univariate level shift model of $\mathrm{Lu}$ and Perron (2008) to the multivariate case, with the persistence of each series stemming from a common level shift process. A recent debate in the univariate literature focuses on the possible confusion between true long memory processes and short memory processes with structural changes in levels. It is well known that when a stationary process is contaminated by level shifts, the fractional integration order is positively biased. For example, Perron (1990) points out that structural changes and unit roots can easily be confused. Granger and Hyung 
(2004) show that the long memory property of volatility could be induced spuriously by the presence of structural breaks, possibly stemming from financial crises. The breaks are identified following the procedure outlined in Bai and Perron (2003). On the other hand, as noted by the authors, the presence of clusters in the levels of the process is also a feature of true long memory processes, so their procedure seems to overreject the possibility of true fractional integration. More recently, Ohanissian et al. (2008) propose a test for evaluating the presence of true long memory processes based on their self-similarity property and Dolado et al. (2005) provide a timedomain test for the null hypothesis of $I(d)$ against $I(0)$ plus structural breaks that is based on the same principles as the well-known Dickey-Fuller unit root test. Lu and Perron (2008) present an univariate random shift model for stock volatilities, exploiting a procedure to estimate the probability and the magnitude of the shifts, and showing that the random occurrence of shifts induces spurious long memory, while Perron and Qu (2010) propose a simple test of long memory against mean shifts, that is based on the number of frequencies used in the log-periodogram regression to estimate $d$.

We estimate our common level shift model using the Kalman filter in a generalization to the multivariate case of the univariate level shift technique by Lu and Perron (2008). We consider both an application to the S\&P500 index and a simulation experiment. We show that in a multivariate context, the presence of common shifts induces confusion between true and spurious fractional cointegration, where the term spurious fractional cointegration indicates that the series are spuriously of long memory, and a linear combination of them eliminates the common deterministic trend. To this end, we study both the regression relation between implied and realized volatility, as in Christensen and Nielsen (2006) and Bandi and Perron (2006), and an alternative system representation of Johansen (2008), namely, a fractional vector error correction (FVEC) model that is of interest in its own right, as it captures the different degrees to which implied and realized volatility adjust to shocks to their long run relation, and does so without imposing zero and unit integration orders. The FVEC model allows for a formal test of fractional cointegration, as in Lasak (2009). As suggested by Lasak (2008) and Johansen and Nielsen (2010), the parameters are all estimated jointly by a profile likelihood estimator based on the reduced rank regression of Johansen (1988).

The paper is organized as follows. Section 2 provides the estimation technique of an alternative model setup to account for the presence of persistence in a multivariate framework, considering the presence of a common level shift process with random intensity that drives jointly 
the dynamics of realized and implied volatility. Section 3 introduces the concept of fractional cointegration and the related estimation techniques. Section 4 presents the data and reports the statistical properties of the sample, while section 5 discusses the estimation results for the alternative models. In section 6 we evaluate the performance of the fractional cointegration tests in small samples and according to alternative data generating processes. Section 7 concludes.

\section{Common Level Shifts}

Our purpose is to model the implied-realized volatility as they were generated by a bivariate common shift process and to evaluate the power of parametric fractional cointegration tests, when the true DGP is given by a multivariate short memory process plus a common level shift component. In particular, we extend the model of Lu and Perron (2008) to the following bivariate model

$$
y_{t}=\tau_{t} \iota+c_{t}
$$

where the $c_{t}=\Phi_{1} c_{t-1}+\ldots+\Phi_{p} c_{t-p}+\epsilon_{t}$ is a $\operatorname{VAR}(\mathrm{p})$ term, where $\epsilon_{t} \sim N(0, \Sigma)$ and $\Sigma$ is a $2 \times 2$ covariance matrix; $\iota$ is a $2 \times 1$ vector of ones, and $\tau_{t}$ is the common random shift component

$$
\tau_{t}=\tau_{t-1}+\delta_{t}
$$

with $\eta_{t} \sim N\left(0, \sigma_{\eta}^{2}\right)$ and $\delta_{t}=\pi_{t} \eta_{t}$ where $\pi_{t}$ is a binomial variable that takes value 1 with probability $\nu$, so that if $\pi_{t}=1$ a level shift occurs.

Given this model setup, it is evident that $\beta y_{t} \sim I(0)$ where $\beta=[1,-1]$, that is the difference $y_{1, t}-y_{2, t}=c_{1, t}-c_{2, t}=z_{t} \sim I(0)$. It is then clear that a linear combination of the two series eliminates the common feature, resulting in a short memory process, so that common level shifts can be easily confused with the presence of fractional cointegration. ${ }^{1}$ Despite its similarity with the Markov regime switching models, see Hamilton (1989), this model setup presents more flexibility since it allows for a potentially infinite number of possible regimes, given that the magnitude of the level shifts is drawn from a normal distribution. For example, in Morana (2002) and Morana (2007) the concept of cobreaking is introduced in a Markov switching setup, where the number of regimes is set a priori. The estimation procedure is outlined in Appendix A. This procedure provides the estimates of $\nu, \sigma_{\eta}^{2}$ and $\Sigma$, allowing to disentangle the noisy

\footnotetext{
${ }^{1}$ Mikosch and Starica (2004) derive in closed form the autocorrelation function of an $I(0)$ process with level shifts showing that this is able to mimic the long range dependence of truly long memory processes.
} 
components from the common shift process, that induces persistence to both series. Section 6 discusses the evaluation of the power of the fractional cointegration tests with respect to a common level shift process through a Monte Carlo exercise on the basis of the empirical estimates obtained with the procedure outlined above.

We also provide a selection procedure to identify the level shifts in the multivariate context, based on the estimated probability $\hat{\nu}$. In particular, we provide an extension of the method of Bai and Perron (2003) to the multivariate case where the shift process is restricted to be common. Our method is based on the following algorithm:

1. Calculate the predicted average number of shifts in the sample, $[\hat{\nu} \cdot T]$;

2. Compute the combination of all the possible position of the number of break dates;

3. For each possible combination of shift dates, compute and subtract the mean of each subsample;

4. For each subsample, estimate a $\operatorname{VAR}(p)$ on the demeaned sample;

5. Compute the $\operatorname{VAR}(p)$ residuals for each choice of break dates;

6. Choose the combination of break dates that minimizes the sum of the squared residuals.

\section{Fractional Cointegration}

The concept of cointegration has been widely studied during the last three decades, since the original paper by Granger (1981). Most of the analysis has concentrated on the special case where a linear (or nonlinear) combination of two or more $\mathrm{I}(1)$ variables is $\mathrm{I}(0)$. Tests for $\mathrm{I}(1) / \mathrm{I}(0)$ cointegration are carried out in a regression setup, as proposed by Engle and Granger (1987), or investigating the rank of the cointegration matrix in a system of equations following the Johansen (1991) procedure.

More recently, part of the literature has focused on the possibility of an extension of the concept of cointegration to fractional processes, i.e. integrated of order $d$, with $d>0$. In particular, given $d<\frac{1}{2}, x_{t} \sim I(d)$ if

$$
(1-L)^{d} x_{t}=\epsilon_{t}
$$


where $\epsilon_{t} \sim I(0)$ and the term $(1-L)^{d}$ results in the binomial expansion

$$
(1-L)^{d}=\sum_{i=0}^{\infty} \frac{\Gamma(i-d)}{\Gamma(-d) \Gamma(i+1)} L^{i}
$$

If $d>-\frac{1}{2}$ the process is invertible and possesses a linear (Wold) representation, and if $d<\frac{1}{2}$ it is covariance stationary. For $d<0$, the process is said to be anti-persistent, while for $d>0$ it is persistent and it has long memory. In this context, the term fractional cointegration refers to a generalization of the concept of cointegration, since it allows linear combinations of $I(d)$ processes to be $\mathrm{I}(d-b)$, with $0<b \leq d$. The term fractional cointegration underlies the idea of the existence of a common stochastic trend, that is integrated of order $d$, while the short period departures from the long run equilibrium are integrated of order $d-b . b$ stands for the fractional order of reduction obtained by the linear combination of $I(d)$ variables.

According to the definition in Granger (1986), two (or more) $I(d)$ series are fractionally cointegrated if there exists a linear combination that is $I(d-b)$, with $b \leq d$. Thus the errors are of lower order of fractional integration than the levels. This means that the series share fractionally integrated stochastic trends of different orders $(I(d)$ and $I(d-b))$, and a linear combination eliminates the largest. A typical situation is when $z_{t}=\left(x_{t}^{\prime}, y_{t}\right) \sim I(d)$ and $e_{t} \sim I(d-b)$ with $d>d-b \geq 0$ in the model

$$
y_{t}=\beta^{\prime} x_{t}+e_{t}
$$

Robinson (1994) shows that OLS estimator of $\beta$ is inconsistent when the errors are fractionally integrated. He introduces in this context the narrow-band frequency domain least squares, a semiparametric method ${ }^{2}$, and proves it is consistent even in situations where the error term is correlated with the regressors. Once that the cointegration vector, $\hat{\beta}_{m}$ has been estimated with respect to the first $m$ frequencies, we look to the estimated fractional integration order of the residuals, $\hat{d}-\hat{b}$, testing whether it is equal to 0 , meaning that the fractional reduction is complete and $d=b$.

The regression approach to the implied-realized volatility relation is focused on the specification

$$
R V_{t}=\alpha+\beta I V_{t}+e_{t} \quad t=1, \ldots, T
$$

where $R V_{t}$ is realized volatility and $I V_{t}$ implied volatility. Unbiasedness of the implied volatility

\footnotetext{
${ }^{2}$ See Appendix B for a description of this estimation method.
} 
forecast corresponds to the hypothesis $\beta=1 \cap \alpha=0$. Following Christensen and Nielsen (2006) and Bandi and Perron (2006), the classical OLS estimator cannot be used to test market efficiency, when interpreting the equation (6) as a cointegration relation. In fact, given that $I V_{t}$ and $R V_{t}$ are fractionally integrated, the long run relationship between them cannot be tested using OLS, since the correlation between $I V_{t}$ and the error term introduces a bias term in the estimation of the cointegration vector. Therefore, Christensen and Nielsen (2006) and Bandi and Perron (2006) propose to estimate equation (6) with the narrow-band frequency domain least squares, henceforth $N B L S$, that focuses on frequencies in the neighborhood of zero and provides unbiased estimates of the cointegration vector. Christensen and Nielsen (2006) find that the condition $\beta=1 \cap \alpha=0$ is satisfied when equation (6) is estimated with $N B L S$. They also find that the fractional integration order of residuals is zero, meaning that the linear combination $R V_{t}-I V_{t} \sim I(0)$. Moreover, as pointed out by Bandi and Perron (2006), estimating the linear projection of $R V_{t}$ on $I V_{t}$ with OLS induces correlation between the residuals and $I V_{t}$, given the presence of volatility risk premium, since the expected realized volatility depends linearly on $I V_{t}$ and the risk premium. They also find unbiasedness in the realized-implied volatility relation. On the other hand, Nielsen (2007) proposes a new testing methodology that provides jointly estimates of the integration orders and the cointegration vector in (6) assuming that the cointegration rank is equal to one and the integration order, $d$, of realized and implied volatility is less than $1 / 2$. The estimation method is based on the local Whittle approximation of the likelihood function concentrated with respect to the cointegration vector. Using the same dataset as Christensen and Nielsen (2006), Nielsen (2007) notes that the evidence of long run unbiasedness becomes less clear, when jointly testing the null $\beta=1 \cap d_{e}=0$, where $d_{e}$ is the integration order of the cointegration residuals.

We evaluate the relative performance of the option market in adjusting its forecasts of the future realized volatility, given a shock to the long run equilibrium relation that represents the link between realized and implied volatility. To this end, we model the realized-implied volatility relation according to an alternative parametric setup that explicitly account for the long-run relation implied by the unbiasedness hypothesis. We explore the relationship between realized and implied volatility in terms of fractional cointegration by means of a fractional vector error 
correction models (FVECM),

$$
\begin{aligned}
\Delta^{d} \log R V_{t} & =\alpha_{R}\left(1-\Delta^{b}\right) \Delta^{d-b}\left(\log R V_{t}+\mu+\beta \log I V_{t}\right)+\epsilon_{t}^{R V} \\
\Delta^{d} \log I V_{t} & =\alpha_{I}\left(1-\Delta^{b}\right) \Delta^{d-b}\left(\log R V_{t}+\mu+\beta \log I V_{t}\right)+\epsilon_{t}^{I V}
\end{aligned}
$$

where $\boldsymbol{\epsilon}_{t}=\left(\epsilon_{t}^{R V}, \epsilon_{t}^{I V}\right)$ are assumed to be Gaussian with mean zero and variance $\Omega$ and $\boldsymbol{\alpha}=$ $\left(\alpha_{R}, \alpha_{I}\right)$ and $\boldsymbol{\beta}=(1, \beta)$ are the error correction and cointegration vectors. The core $(1-$ $\left.\Delta^{b}\right) \Delta^{d-b}\left(\log R V_{t}+\beta \log I V_{t}\right)$ defines the cointegration relation, where $d$ represents the fractional integration order of the realized and implied volatilities. There will be full cointegration in the case $d=b$, meaning that the residuals from the cointegration relation are integrated of order 0 . In the FVECM model, the element $\alpha_{i}$ of the vector $\alpha$ measures the single period response of variable $i$ to the shock on the equilibrium relation. In our case, if $\alpha_{i}$ is found to be significant, then the realized (implied) volatility moves to restore the unique long-run relationship implied by the unbiasedness hypothesis. In addition, the FVECM allows for a flexible characterization of the cointegration relation, where the integration orders of the endogenous variables, $d$, and the cointegration residuals, $d-b$, are not restricted to assume values 1 and 0 , respectively. This model presents several advantages over the traditional cointegration regression; in fact

- The integration order of the endogenous variables and the fractional cointegration order are defined by two parameters $d$ and $b$, with $0<b \leq d$, that are jointly estimated;

- It allows for a formal test of fractional cointegration based on the cointegration rank, as in Lasak (2009);

- The specification of the model makes it an instrument for evaluating the dynamic response of a variable to a shock to an other variable in the system;

- The vector of loading parameters $\alpha$ gives a measure of the speed of adjustment to the long run equilibrium given a short run departure from that, allowing us to draw conclusions on the relative efficiency of the option market;

- As suggested in Lasak (2008) and Johansen and Nielsen (2010), the parameters are all estimated jointly by a profile likelihood estimator based on the reduced rank regression of Johansen (1988). This estimation procedure differs from the two step procedure implemented by Christensen and Nielsen (2006) and Bandi and Perron (2006), and we consider both in the following. 
- In general notation, the system representation of Johansen $(2008)^{3}$ is

$$
\Delta^{d} X_{t}=\left(1-\Delta^{b}\right)\left(\Delta^{d-b} \alpha \beta^{*^{\prime}} Y_{t}\right)+\sum_{j=1}^{K} \Gamma_{j} \Delta^{d} L_{b}^{j} X_{t}+\epsilon_{t}
$$

and in the present case $Y_{t}=\left[\log R V_{t}, \log I V_{t}, 1\right]^{\prime}$ and $\beta^{*^{\prime}}=[1, \beta, \mu]$, so that $R V_{t}$ and $I V_{t}$ are fractionally cointegrated if $\operatorname{rank}\left(\Pi^{*}\right)=\operatorname{rank}\left(\alpha \beta^{* \prime}\right)=1$. As pointed out by Johansen (2008), when $\beta^{\prime} \alpha=-I_{r}$, then model (7) corresponds to the triangular system studied in Breitung and Hassler (2002), so that we have a representation in terms of common trends factors and equilibrium errors. A corresponding representation in terms of cointegrated FIVAR, see Duecker and Startz (1998) for an application, is not yet available. Assuming that the cointegration rank, $r$, is known already, model (7) is estimated following the procedure outlined in Lasak (2008). Lasak (2008) considers the fractional reduction of integration order, $b$, in non-stationary systems. In particular, the model (7) is estimated via a maximum likelihood technique analogous to that developed by Johansen (1991) for the standard VECM, where $d$ and $b$ are restricted to be equal to 1 . Details on this estimation method are in Appendix C. The asymptotic distribution of the FVECM estimators are studied in Lasak (2008) and Johansen and Nielsen (2010), while this estimation procedure has been employed by Rossi and Santucci de Magistris (2009), who show finite sample properties of the estimators through a Monte Carlo exercise.

\section{Data}

Our dataset consists of options prices on monthly future contracts on S\&P 500, from April 1988 until October 2007. For each month, we sample the initial and the expiring date of the option contract. In particular, since the option contracts expire on the Friday immediately preceding the second Saturday of each month, we select the Tuesday just after the expiration date as initial date for the next monthly contract ${ }^{4}$. In this way, we avoid the presence of overlapping observations and the possible bias induced by the transaction on the first Monday just after the delivery of the new contract.

The (annualized) monthly implied volatility, $I V_{t}$, is then calculated on a traded at-the-money

\footnotetext{
${ }^{3}$ Johansen (2008) presents an alternative parametrization, with respect to that suggested by Granger (1986). This model setup allows for Granger representation, based on the new lag operator $L_{b}=1-(1-L)^{b}$. On the other hand, the error correction relation is the same in both Granger (1986) and Johansen (2008).

${ }^{4}$ Business holidays may modify this sampling rule.
} 
option contract with one month to maturity. In particular, we employ the well known Black and Scholes (1973) formula, where the no arbitrage price, $C$, of an European call is given by

$$
\begin{aligned}
C(F, K, \tau, r, \sigma) & =e^{-r(\tau+\Delta)}[F \Phi(\delta)-K \Phi(\delta-\sigma \sqrt{\tau}) \\
\delta & =\frac{\ln (F / K)+\left(\frac{1}{2} \sigma^{2}\right) \tau}{\sigma \sqrt{\tau}}
\end{aligned}
$$

where $F$ is the spot price of the underlying asset, $K$ the strike price, $r$ is the riskless interest rate, $\Phi($.$) is the standard normal c.d.f., and \sigma$ is the volatility of the underlying asset. The monthly implied volatility is recovered, given the observed price of the option, $C_{t}^{o}$, inverting (8) and solving numerically with respect to $\sigma$. Repeating this procedure for every period $t$, it will result in the series of implied volatility, $I V_{t}$, for each month $t \in[1, T]$.

Since our dataset contains also the intradaily futures prices on S\&P 500, we are able to calculate a precise ex-post measure of the volatility observed on the underlying futures contract during month $t$. Following Bandi and Perron (2006), the realized (annualized) monthly volatility measure of the futures on S\&P 500 is calculated as

$$
R V_{t}=\sqrt{\frac{1}{M_{t}} \sum_{j=1}^{M_{t}} \sigma_{j, R V}^{2} \times 252}
$$

where $\sigma_{j, R V}$ is the $j$-th daily realized volatility on $\mathrm{S} \& \mathrm{P} 500$ futures contract in month $t$ while $M_{t}$ is the number of trading day in the month $t$, that is the number of trading days between the delivery and the expiration of the option contract; $R V_{t}$, then, measures the average annualized realized volatility in month $t$. The daily realized volatility is calculated, following Andersen et al. (2001b), as

$$
\sigma_{j, R V}^{2}=\sum_{k=1}^{N_{j}} r_{j, k}^{2}
$$

where $r_{j, k}$ is the five minute return on future price, while $N_{j}$ is the number of intradaily returns. High frequency five minutes returns are the basis for the daily realized volatility, so that $N_{j}=72$.

We extend our analysis, considering the observations on VIX, hence after $V I X_{t}$, implied volatility of options on S\&P 500 traded at the Chicago Board Option Exchange (CBOE), from January 1990 until March 2007. The VIX is the (annualized) implied volatility of a nontraded (synthetic) at-the-money option contract with one month to maturity. This measure is less affected by the problems that affect standard implied volatility measures, such as the potential nonsynchronous 
measurement of option and index levels, early exercise and dividends, bid-ask spreads.

We use monthly non-overlapping observations by taking the closing value of each month, and multiply the VIX data by a constant factor equal to $\sqrt{\frac{252}{365}}$ to account for the difference between trading days and calendar days in a year.

Given the time series of daily spot prices of the S\&P 500, we propose an alternative measure for the monthly realized volatility that is the Realized Range, $R R V_{t}$, that is defined as

$$
R R V_{t}=\sqrt{\frac{1}{M_{t}} \sum_{j=1}^{M_{t}} \sigma_{j}^{2} \times 252} \quad t=1, \ldots, T
$$

where $n_{t}$ is the number of trading days in month $t$ and

$$
\sigma_{j}^{2}=\lambda^{-1}\left(P_{j}-p_{j}\right)^{2}+\left(o_{j}-c_{j-1}\right)^{2}
$$

where $\lambda=4 \log (2)$, is the Parkinson (1980) estimator of the daily volatility, that is defined as the difference between the highest, $P_{j}$, and lowest, $p_{j}$, log prices of the S\&P 500 on the $j-t h$ day of month $t$ plus the difference between the log opening price, $o_{j}$, and the log closing price, $c_{j-1}$, of the day before. Christensen and Podolskij (2007) have investigated the efficiency of the range estimator, noting that daily range is more efficient than the daily squared returns, corresponding to the realized volatility calculated on the basis of 2-3 hours returns.

Our dataset then includes 234 non-overlapping monthly measures of annualized realized volatility and implied volatility on futures of S\&P 500 and 207 non-overlapping monthly measures of annualized realized range and implied volatility of spot prices of S\&P 500. Figure 1 plots the dynamic behavior of the logarithm of the series under analysis. From a visual inspection, it appears evident that implied and realized volatility follows similar dynamic patterns, and are linked together. In particular, we observe the contemporaneous presence of periods of high and low volatility, a characteristic of both long memory and level shift processes. Table 1 reports some sample statistics for $I V_{t}, R V_{t}, V I X_{t}$ and $R R V_{t}$. Implied volatility is, on average, higher than realized volatility, reflecting the possible presence of a risk premium in the determination of option prices, see Bollerslev et al. (2009). On the other hand, realized measures are, as expected, more volatile than implied volatility, since the latter is an expectation of the volatility during next month. The high positive skewness is caused by the fact that volatilities are restricted to be positive, so that we are more likely to observe positive deviations from the mean. Since the 
$\log$ of realized volatility is closer to be normal, we show the sample statistics of the logarithm of volatility measures in table 1. As highlighted by Andersen et al. (2001a), the logarithmic transformation reduces drastically the level of excess kurtosis and skewness is also close to 0 . For this reason, we refer to logarithm of volatility in the rest of the paper.

We first evaluate and compare the degree of fractional integration of implied and realized volatilities. Therefore, we propose a semiparametric test on the equality of the degree of fractional integration of realized and implied volatilities, applying a multivariate extension of the exact local Whittle estimator used in a univariate setup by Shimotsu and Phillips (2005). Nielsen and Shimotsu (2007) propose, as in Robinson and Yajima (2002), a test statistic for the equality of integration orders that, in the bivariate case, is

$$
\widehat{T}_{0}=m_{d}(S \hat{d})^{\prime}\left(S \frac{1}{4} \hat{D}^{-1}(\hat{G} \odot \hat{G}) \hat{D}^{-1} S^{\prime 2}\right)^{-1}(S \hat{d})
$$

where $\odot$ denotes the Hadamard product, $S=[1,-1]^{\prime}, h(T)=\log (T)^{-k}$ for $k>0, D=$ $\operatorname{diag}\left(G_{11}, G_{22}\right)$, while $\hat{G}=\frac{1}{m} \sum_{j=1}^{m} \operatorname{Re}\left(I_{j}\right)$. The parameter vector $\hat{d}$ is the exact Local Whittle estimator of $d$, introduced by Shimotsu and Phillips (2005). Table 2 shows the results of the semiparametric analysis of the fractional integration order of realized-implied volatilities. The bandwidth parameters are $T^{0.7}, T^{0.6}$ and $T^{0.5}$. We notice that implied volatility presents an higher degree of fractional integration with respect to the realized volatilities, even if the $\widehat{T}_{0}$ statistic takes high values only in the case of volatilities on future contracts. Since the $95 \%$ critical value of a $\chi_{1}^{2}$ is 3.841 , we cannot reject the null of equality of the integration orders in most cases, so that we can conclude that monthly implied and realized volatility share the same order of fractional integration. The estimates of the fractional integration order, $d$, are in all cases in the non-stationary region, but they are all inferior to the unit value. This evidence contrasts with the findings in Andersen et al. (2001a), where realized volatility is stationary with fractional integration order equal to 0.4 . This fact is perhaps due to the aggregation of the series over a monthly horizon, that increases the level of persistence. Granger and Hyung (2004) point out the idea that the excess persistence in volatility series could be induced spuriously by the presence of occasional structural breaks. For this reason, we implement the tests proposed by Shimotsu (2006), that are based on sample splitting and $d$-differencing, in order to evaluate whether long memory is a true feature of the data. ${ }^{5}$ Table 3 reports the results of the testing

\footnotetext{
${ }^{5}$ We refer to the paper by Shimotsu (2006) for a careful description of the estimation techniques.
} 
procedures. From table 3 is clear that tests based on sample splitting and $d$-differencing cannot refuse in all cases the null hypothesis of true long memory. ${ }^{6}$. Moreover, we evaluate the presence of long memory, according to the testing procedure outlined in Ohanissian et al. (2008), that is based on temporal aggregation, exploiting the self-similarity of truly long memory processes. The test strongly rejects the null of truly long memory for both realized and implied volatility. Given these results, it is worth exploring the possibility of alternative setup for long run dependence, as outlined in section 2 .

\section{Empirical results}

First we implement the analysis of the cointegration regression of $\log R V_{t}$ on $\log I V_{t}$, that contains all the information available up to $t$, that is incorporated in the determination of the option price at time $t$. In particular, we implement the FDLS analysis as outlined in section 2. Table 4 reports the results of the cointegration analysis and confirms the findings of Bandi and Perron (2006). The integration order of the cointegration residuals is, in most cases, non significantly different from 0 , suggesting that the stochastic trend between implied and realized volatility determines their joint long run dynamics. Moreover, we confirm the long run unbiasedness hypothesis since, when using FDLS, $\beta$ is statistically equal to 1 and $\alpha$ is equal to zero. ${ }^{7}$

The analysis of fractional cointegration is done also in terms of the cointegrated system (7). ${ }^{8}$ The system is estimated without any lag of the dependent variables on the right hand side, and results are reported in table 5. The results in terms of fractional cointegration are similar to those obtained with the regression setup. The rank tests reject the null that the cointegration rank is zero, so that the series are fractionally cointegrated. Interestingly, both for futures and spot volatility, we find that $b$ is lower than $d$, meaning that the cointegration residuals have long memory. In particular, the difference $\hat{d}-\hat{b}$ is significantly different from zero and close to 0.25 , so that we can conclude that the stochastic trend between implied and realized volatility accounts only partially in determining their common long run dynamics. Furthermore, the fact, that operators on financial markets commit a forecast error with long memory, contrasts with the rational expectation hypothesis, see Davidson et al. (2006).

\footnotetext{
${ }^{6}$ This evidence seems to be also confirmed by a visual inspection of the graph of the log-periodogram estimation of $d$ with respect to $m_{d}$, as suggested by Perron and $\mathrm{Qu}(2010)$. In fact, the pattern of the log-periodogram estimates of $d$ appears comparable to that of a long memory process plus level shifts, see Perron and Qu (2010, p. 285).

${ }^{7}$ As in Bandi and Perron (2006), the confidence intervals are calculated by means of a subsampling techique.

${ }^{8} \mathrm{~A}$ lag selection procedure, based on the Schwarz Criterion, indicates that no short term dynamics have to be included in the Johansen (2008) model, see equation 7.
} 
The rational expectation hypothesis, in fact, implies that ex-post and ex-ante volatilities differ only by a random $I(0)$ error term, that is interpreted as an idiosyncratic deviation from the long run equilibrium implied by the cointegration relation. In this sense, deviations from equilibrium in time $t$ should be unpredictable based on information up to time $t-1$, which implies $d=b$. When $b<d$, the forecast error has long memory and it is affected by past forecast errors. Therefore, operators have limited rationality, since they gradually learn to exploit the information contained in the present and past errors. The adjustment parameters, $\alpha_{R}$ and $\alpha_{I}$, are different from zero and with the expected signs. However, $\alpha_{R}$ in the future case is not significant. In this sense, the ex-post realized volatility of future contracts leads the implied volatility toward the long run equilibrium, and this additional information is incorporated in model (7). In fact, all the corrections toward the long run equilibrium are done by the implied volatility, meaning that operators on financial markets weight the present and past disposable information to produce forecast of the future realized volatility. For what concerns the unbiasedeness of futures contracts, $\beta$ is close to theoretical value 1 , that is included in the bootstrapped confidence interval and and $\mu$ is significantly different from zero, suggesting the presence of a significant volatility risk premium, that is relevant in the fractional cointegration relationship.

We also provide an estimate of the model (1) in Panel A of table 6, following the procedure outlined in section 3 . The probability of a level shift is around $3-4 \%$, meaning that we should observe an average of $6-8$ shifts in the sample. The standard deviation of the level shift, $\sigma_{\eta}$, is approximately 3 times larger than the standard deviation of the residuals. This finding highlights the importance of the level shifts in accounting for the common persistence of the two series, and reinforces the idea that the common shift process induces fractional cointegration, since the persistence of the series is removed by a linear combination of realized and implied volatility. Panel B reports the estimates of the common level shift model, where the short run dynamics are described by a VAR(1) process. This setup is in accordance with the findings in Lu and Perron (2008) and Perron and Qu (2010), that are consistent with a short memory process with level shifts. In particular, we notice a strong reduction of the probability $\nu$ with respect to the white noise case, so that we observe an average of 2-3 shifts in both samples. Moreover, the standard deviation of the shifts is reduced, but it is still higher than the standard deviation of the residuals. This suggests that, including some short run dynamics in model (1) reduces the impact of the level shifts since the persistence of the series is partially accounted for by the autoregressive components. 
For robustness purposes, we verify the presence of a single common level shift, allowing the magnitudes of the shifts to be non-perfectly correlated. In particular, we modify (19), assuming $\eta_{1, t} \sim N\left(0, \Sigma_{\eta}\right)$ where

$$
\Sigma_{\eta}^{\rho}=\left[\begin{array}{cc}
\sigma_{\eta}^{2} & \rho \sigma_{\eta}^{2} \\
\rho \sigma_{\eta}^{2} & \sigma_{\eta}^{2} \\
&
\end{array}\right]
$$

with $\rho \in[-1,1]$. in this case, $\eta_{1, t}$ is a bivariate vector, and $\Sigma_{\eta}^{\rho}$ replaces $\Sigma_{\eta}=\sigma_{\eta}^{2} \iota \iota^{\prime}$ in the Kalman filter. When $\rho=1$, then the level shift process is exactly the same across the volatility series. We then test for the restriction $\rho=1$ by means of a Wald test. $W^{\rho}$ in table 6 is the Wald test for the restriction $\rho=1^{9}$. Especially when including VAR dynamics, we cannot reject the null when we allow for short run dynamics $\operatorname{VAR}(1)$, so that the hypothesis of an unique common level shift process is compatible with the data. Consistently with the estimates of model (1), we then identify the break dates on the basis of the estimated probability $\nu$ according to the identification procedure outlined in section 3. Figure 2 shows the volatility series with the identified shift process based on $\hat{\nu}$ from Panel B of table 6. We notice that volatilities of future contracts are characterized by 3 level shifts and are particularly high between the 1996 and 2002, while spot volatilities are not significantly low at the beginning of the 90s. As in Lu and Perron (2008), the point estimate imply few shifts.

Table 7 reports the parameter estimates of a $\operatorname{VAR}(1)$

$$
X_{t}=\gamma+\Phi_{1} X_{t-1}+z_{t}
$$

where $X_{t}=\left[\log R V_{t}, \log I V_{t}\right]$, or $X_{t}=\left[\log R R V_{t}, \log V I X_{t}\right]$, computed on the original series of realized and implied volatility and on the series after removing the common shift process. Once the common deterministic trend or level shift series is accounted for, implied volatility does not contain any explanatory power for the next period realized volatility, thus confirming the findings based on the FVECM representation. In contrast to the results of the $\operatorname{VAR}(1)$ on the original series in panel $\mathrm{A}$, the parameter $\phi_{12}$ in the panel B is no longer significant at $5 \%$. Thus, when the common long run dynamics are disentangled from the short run dynamics, whether in the FVECM or level shift model, implied volatility does not retain predictive information about future volatility. We conclude that realized volatility represents the driving force of the

\footnotetext{
${ }^{9}$ Since $\rho=1$ is on the boundary of the admissible values for $\rho$, the Wald test at level $\alpha$ is compared with the $(1-2 \alpha)$-quantile of a $\chi_{1}^{2}$
} 
entire system. Implied volatility adjusts to realized volatility, and it does pick up the long run component of the latter, thus explaining the received evidence on the forecasting power of implied volatility.

\section{Simulations}

Given the estimates of the FVECM and the level shift model, we run a Monte Carlo simulation in order to evaluate the size and the power of the fractional cointegration tests in finite samples when the data are generated by alternative data generation processes. In particular, in order to evaluate the size of the fractional cointegration tests, we simulate pseudo data under the null of no cointegration from the following DGPs,

- Fractional Cointegration system with $\boldsymbol{\alpha}=0$ :

$$
\begin{aligned}
\Delta^{d} Y_{t} & =\epsilon_{t}^{y} \\
\Delta^{d} X_{t} & =\epsilon_{t}^{x}
\end{aligned}
$$

- Level shift with two independent shift processes, $\tau_{t}^{1}$ and $\tau_{t}^{2}$ :

$$
\begin{aligned}
Y_{t} & =\tau_{t}^{1}+\epsilon_{t}^{y} \\
X_{t} & =\tau_{t}^{2}+\epsilon_{t}^{x}
\end{aligned}
$$

- Cointegration regression with no reduction of the integration order:

$$
\begin{aligned}
& X_{t}=\Delta^{-d} u_{t} \\
& Y_{t}=\beta X_{t}+\Delta^{-d} \epsilon_{t}
\end{aligned}
$$

where $\boldsymbol{\epsilon}_{t}=\left(\epsilon_{t}^{y}, \epsilon_{t}^{x}\right)$ are distributed as a bivariate normal with variance $\hat{\Omega}$, set at empirical estimates that did not impose the null of no cointegration. The binomial level shift processes in (17), say $\tau_{1}$ and $\tau_{2}$, are independent with the same probability $\nu$, and shift variance $\sigma_{\eta}^{2} \cdot{ }^{10}$ Note that, given the presence of two distinct level shift processes in (17), the resulting series are no more fractionally cointegrated; in fact, any linear combination between $y_{t}$ and $x_{t}$ will not result

\footnotetext{
${ }^{10}$ The pseudo series are simulated according to the estimates for the implied and realized volatility obtained in section 4 .
} 
in a $I(0)$ process, except in the case where $\tau_{1}=\tau_{2}$ that happens with probability 0.

Table 8 reports the empirical size of the cointegration tests for 1000 Monte Carlo replications. We notice that, when the data are generated according to models (16) and (18) the $\lambda_{\max }$ test is not distorted, while the $t$-test based on the cointegration regression overrejects the null. On the other hand, we notice that the empirical size of the cointegration tests, when data are generated according to a bivariate independent level shift process, is upwardly biased, in particular when implementing the test on the cointegration rank. The empirical rejection rate increases dramatically when adding $\operatorname{VAR}(1)$ dynamics, so that we clearly address this high distortion to the VAR component, that dominates the dynamics of the system. We conclude that, when the true process is a highly persistent $\operatorname{VAR}(1)^{11}$ and the errors are correlated, the usual cointegration tests indicate the presence of cointegration even when it is not there. For a related point in a situation without level shifts, Gonzalo and Lee (1998) found that a persistent $\operatorname{VAR}(1)$ creates the false impression of long memory.

The power of the cointegration tests is evaluated simulating according to the bivariate random level shift (1) with a common shift process and to the FVECM model (7). The parameters used in the simulation are those estimated in section 4 . Table 9 reports the percentage rejection of the null given that the null is false. The rejection rate is generally very high under both the simulation schemes. This shows that findings of fractional cointegration may be accounted for by common level shifts, since the null of absence of fractional cointegration is rejected in most cases, even if the data are generated according to a common level shift process. Moreover, we evaluate the power of the test for alternative values of $\alpha$ and $\sigma_{\eta}$, see table 10 . The power of the regression based test decreases faster than that of the rank test when reducing the value of the parameters $\alpha_{1}$ and $\alpha_{2}$ in the FVECM setup. Similar results are obtained when simulating under the level shift model, reducing the variance of the shifts. Given the results of this Monte Carlo experiment, we conclude that the rank test based on FVECM representation correctly detect fractional cointegration when data are generated under FVECM or according to a cointegration regression setup. The parametric rank test is highly size distorted when the true DGP is a common level shift process. Finally, the rejection rate of the rank test is very high under common level shifts, so that findings of persistence in volatility and fractional cointegration may be due to common level shifts.

\footnotetext{
${ }^{11}$ In this case, one of the eigenvalues of the autoregressive matrix is close to 1
} 


\section{Conclusions}

We consider the possibility that the observed persistence of realized and implied volatility, and the strong relation between the two, is due to a common level shift process. We carry out a new multivariate estimation technique, so that we obtain the probability of the occurrence of a common level shift and its standard deviation. We find that shifts are very likely to happen, and their standard deviation accounts for the overall variance of the series and their persistence. Once the underlying common feature is removed, implied volatility does not have any predictive power for realized volatility. This evidence is in accordance with the findings obtained in the fractional cointegration framework and provides additional insights on the information content of option prices. Essentially, realized volatility drives the dynamics of the system, and implied volatility adjusts to realized volatility, picking up the long run component and acting as an efficient forecast. In a Monte Carlo setup, we show that the usual fractional cointegration tests display high probabilities of detecting fractional cointegration when the true DGP is a common level shift model. Thus, the empirical evidence that has been interpreted as long memory in volatility and fractional cointegration is accounted for by our common level shift model.

\section{A Common shifts estimation method}

The model 1 is then written in a state-space form, where the innovations to the common shift component $\tau_{t}$ are distributed according to a mixture of normal distributions

$$
\delta_{t}=\pi_{t} \eta_{1 t}+\left(1-\pi_{t}\right) \eta_{2 t}
$$

where $\eta_{k, t} \sim$ iid $N\left(0, \sigma_{\eta_{k}}^{2}\right)$. In order to recover model $(1)$, we set $\sigma_{\eta_{1}}^{2}=\sigma_{\eta}^{2}$ and $\sigma_{\eta_{2}}^{2}=0$, and rewrite in terms of first differences

$$
\Delta y_{t}=c_{t}-c_{t-1}+\iota \delta_{t}
$$

or in the corresponding state-space form

$$
\begin{aligned}
\Delta y_{t} & =H X_{t}+\iota \delta_{t} \\
X_{t} & =F X_{t-1}+U_{t}
\end{aligned}
$$


where $\iota$ is a $2 \times 1$ vector of ones, $H$ is a $2 \times 4$ matrix, whose rows select the elements of $X_{t}$, so that $H_{1}=[1,0,-1,0]$ and $H_{2}=[0,1,0,-1] . X_{t}$ is a $4 \times 1$ vector that stacks the state variable $c_{k, t}$ in a column vector so that $X_{t}=\left[c_{1, t}, c_{2, t}, c_{1, t-1}, c_{2, t-1}\right]$. The transition matrix in the state equation, $F$, is a $4 \times 4$ block matrix of zeros, where the lower extra diagonal block is a $2 \times 2$ identity matrix, while $U_{t}$ is a $4 \times 1$ vector of innovations $U_{t}=\left[c_{1, t}, c_{2, t}, 0,0\right]$ with a $4 \times 4$ block diagonal variance and covariance matrix $Q$ of zeros, with the upper $2 \times 2$ diagonal block equal to $\Sigma$. Considering a VAR(1) specification for the short run dynamics, we simply have to impose a transition matrix of the form

$$
F_{i}=\left[\begin{array}{cccc}
\phi_{11} & \phi_{12} & 0 & 0 \\
\phi_{21} & \phi_{22} & 0 & 0 \\
1 & 0 & 0 & 0 \\
0 & 1 & 0 & 0
\end{array}\right] \quad i=1,2
$$

The state-space model is estimated with the Kalman-filter technique, that is used to generate the log-likelihood function, given the realization of the latent state variable. The estimation procedure extends the $\mathrm{Lu}$ and Perron (2008) to the bivariate case, so that we remand the reader to their paper ${ }^{12}$. In the multivariate case outlined above, the conditional Gaussian likelihood function of $\Delta y_{t}$ is equal to

$$
f\left(\Delta y_{t} \mid s_{t-1}=i, s_{t}=j, Y_{t-1} ; \theta\right)=\frac{1}{2 \pi}\left|f_{t}^{(k, j)}\right|^{-\frac{1}{2}} \exp \left\{-\frac{v_{t}^{(i j)}\left(f_{t}^{(i, j)}\right)^{-1} v_{t}^{(i j)}}{2}\right\}
$$

where $s_{t}=1$ when $\pi_{t}=1$, so that a level shift occurs, and $i$ and $j$ may assume both 0 and 1 value. $v_{i, t}^{(i j)}=\Delta y_{t}-\Delta y_{t \mid t-1}^{(i)}$ is the prediction error, while $f_{t}^{(k, j)}=E\left[v_{t}^{(i j)} v_{t}^{\left(i j^{\prime}\right)}\right]$ is the $2 \times 2$ prediction error variance. The prediction error is calculated as the difference between $\Delta y_{t}$ and $H X_{i, t \mid t-1}$ that is the best forecast of the state variable conditional on all the information at time $t-1$,

$$
X_{t \mid t-1}=F X_{t-1 \mid t-1}^{i}
$$

with associated variance

$$
P_{t \mid t-1}=F P_{t-1 \mid t-1}^{i} F^{\prime}+Q
$$

\footnotetext{
${ }^{12}$ We maintain the notation as close as possible to Lu and Perron (2008)
} 
so that

$$
f_{t}^{(i, j)}=H P_{t-1 \mid t-1}^{i} H^{\prime}+R_{j}
$$

where $R_{j}=\sigma_{\eta}^{2} \iota \iota^{\prime}=\Sigma_{\eta}$ with probability $\nu$, and $R_{j}=0$ with probability $1-\nu$. The log likelihood function is then defined as

$$
\ln (L)=\sum_{t=1}^{T} f\left(\Delta y_{t} \mid Y_{t-1} ; \theta\right)
$$

with

$$
\begin{aligned}
f\left(\Delta y_{t} \mid Y_{t-1} ; \theta\right) & =\sum_{k=0}^{1} \sum_{j=0}^{1} f\left(\Delta y_{t} \mid s_{t-1}=k, s_{t}=j, Y_{t-1} ; \theta\right) \operatorname{Pr}\left(s_{t-1}=k, s_{t}=j, Y_{t-1} ; \theta\right) \\
& \equiv \mathbf{1}\left(\hat{\xi}_{t \mid t-1} \odot \omega_{t}\right)
\end{aligned}
$$

where $\hat{\xi}_{t \mid t-1}$ is a $4 \times 1$ vector of conditional probabilities, whose $(i, j)$ elements are $\operatorname{Pr}\left(s_{t-1}=\right.$ $\left.k, s_{t}=j, Y_{t-1} ; \theta\right)$, while $\omega_{t}$ is the $4 \times 1$ vector of the conditional densities $f\left(\Delta y_{t} \mid s_{t-1}=k, s_{t}=\right.$ $\left.j, Y_{t-1} ; \theta\right)$. The evolution of $\hat{\xi}_{t \mid t-1}$ is given by

$$
\left[\begin{array}{l}
\hat{\xi}_{t+1 \mid t}^{11} \\
\hat{\xi}_{t+1 \mid t}^{01} \\
\hat{\xi}_{t+1 \mid t}^{10} \\
\hat{\xi}_{t+1 \mid t}^{00}
\end{array}\right]=\left[\begin{array}{cccc}
\nu & \nu & 0 & 0 \\
0 & 0 & \nu & \nu \\
(1-\nu) & (1-\nu) & 0 & 0 \\
0 & 0 & (1-\nu) & (1-\nu)
\end{array}\right]\left[\begin{array}{c}
\hat{\xi}_{t \mid t}^{11} \\
\hat{\xi}_{t \mid t}^{01} \\
\hat{\xi}_{t \mid t}^{10} \\
\hat{\xi}_{t \mid t}^{00}
\end{array}\right]
$$

or more compactly

$$
\hat{\xi}_{t+1 \mid t}=\Pi \hat{\xi}_{t \mid t}
$$

where $\hat{\xi}_{t \mid t}$ is given by

$$
\hat{\xi}_{t \mid t}=\frac{\left(\hat{\xi}_{t \mid t-1} \odot \omega_{t}\right)}{\mathbf{1}\left(\hat{\xi}_{t \mid t-1} \odot \omega_{t}\right)}
$$

while the update for the state variable is given by

$$
X_{t \mid t}^{i j}=X_{t \mid t-1}^{i}+P_{t \mid t-1}^{i} H^{\prime}\left(H P_{t \mid t-1}^{i} H^{\prime}+R_{j}\right)^{-1}\left(\Delta y_{t}-H X_{t \mid t-1}^{i}\right)
$$

and its conditional variance

$$
P_{t \mid t}^{i j}=P_{t \mid t-1}^{i}-P_{t \mid t-1}^{i} H^{\prime}\left(H P_{t \mid t-1}^{i} H^{\prime}+R_{j}\right)^{-1} H P_{t \mid t-1}^{i}
$$


so that

$$
X_{t \mid t}^{j}=\frac{\sum_{i=0}^{1} \hat{\xi}_{t \mid t}^{i j} X_{t \mid t}^{i j}}{\sum_{i=0}^{1} \hat{\xi}_{t \mid t}^{i j}}
$$

and, finally

$$
P_{t \mid t}^{j}=\frac{\sum_{i=0}^{1} \hat{\xi}_{t \mid t}^{i j}\left[P_{t \mid t}^{i j}+\left(X_{t \mid t}^{j}-X_{t \mid t}^{i j}\right)\left(X_{t \mid t}^{j}-X_{t \mid t}^{i j}\right)^{\prime}\right]}{\sum_{i=0}^{1} \hat{\xi}_{t \mid t}^{i j}}
$$

\section{B Frequency domain least squares}

Robinson and Marinucci (2003) and Marinucci and Robinson (2001) show that these semiparametric estimators are consistent for general orders of fractional integration $d$ for the individual series and $d-b$ for the errors in the cointegrating relation and for arbitrary short run dynamics. Define the discrete Fourier transform of an observed vector $\left\{a_{t}, t=1, \ldots, T\right\}$

$$
w_{a}(\lambda)=\frac{1}{\sqrt{2 \pi T}} \sum_{t=1}^{T} a_{t} \mathrm{e}^{i t \lambda}
$$

If $\left\{b_{t}, t=1, \ldots, T\right\}$ is an another observed vector, the cross periodogram matrix between $a_{t}$ and $b_{t}$ is

$$
I_{a b}(\lambda)=w_{a}(\lambda) w_{b}^{*}(\lambda)=I_{a b}^{c}(\lambda)+\mathrm{i} I_{a b}^{q}(\lambda)
$$

where the asterisk is the transposed complex conjugation, and $c, q$ indicate the co- and quadrature periodogram, respectively. The discretely averaged co-periodogram is

$$
\widehat{F}_{a b}(k, l)=\frac{2 \pi}{T} \sum_{j=k}^{l} I_{a b}^{c}\left(\lambda_{j}\right), \quad 1 \leq k \leq l \leq T-1
$$

for $\lambda_{j}=2 \pi j / T$. Thus we obtain the frequency domain least squares estimator

$$
\widehat{\beta}_{m}=\widehat{F}_{x x}^{-1}(1, m) \widehat{F}_{x y}(1, m)
$$

of $\beta$ in regression (5). If

$$
\frac{1}{m}+\frac{m}{T} \rightarrow 0 \quad \text { as } \quad T \rightarrow \infty
$$


then $\widehat{\beta}_{m}$ is called a narrow-band FDLS estimator, since it uses only a degenerating band of frequencies around the origin. Robinson and Marinucci (2003) show that

$$
\widehat{\beta}_{m}-\beta=O_{p}\left(\left(\frac{T}{m}\right)^{-b}\right), \quad \text { as } \quad T \rightarrow \infty .
$$

Under fractional cointegration $d_{e}<\min \left(d_{i}\right)$, so the estimator $\widehat{\beta}_{m}$ are consistent for $\beta$. Moreover, if the integration order of the raw data series is common, the stochastic order of magnitude of the estimator varies with the strength of the cointegrating relation $b$.

Christensen and Nielsen (2006) have derived the asymptotic distribution of $\hat{\beta}_{m}$ when $0<d<\frac{1}{2}$ and $0<b \leq d$. In particular, in the simple case of two variables, this is find to be equal to

$$
\sqrt{m} \lambda_{m}^{b}\left(\hat{\beta}_{m}-\beta\right) \rightarrow N\left(0, \frac{g_{e}\left(1-2 d_{x}\right)^{2}}{2 g_{x}\left(1-2 d_{x}-2 d_{e}\right)}\right)
$$

where $g_{e}$ and $g_{x}$ correspond to $\operatorname{var}\left(\Delta^{b} e_{t}\right)$ and $\operatorname{var}\left(\Delta^{d} x_{t}\right)$.

\section{FVECM estimation method}

Define $Z_{0, t}=\Delta^{d} X_{t}$ and $Z_{1, t}=\left(\Delta^{d-b}-\Delta^{d}\right) X_{t}$ and $Z_{k, t}=\Delta^{d} L_{b}^{k} X_{t}$ for $k=1, \ldots, K$, and the system (7) can be written as

$$
Z_{0, t}=\alpha \beta^{\prime} Z_{1, t}+\Gamma Z_{k, t}+\epsilon_{t}
$$

where $\Gamma=\left(\Gamma_{1}, \ldots, \Gamma_{k-1}\right)$. so that the residual of the regression of $Z_{0, t}$ and $Z_{1, t}$ on $Z_{k, t}$ can be defined as

$$
R_{i, t}=Z_{i, t}-M_{i k} M_{k k}^{-1} Z_{k, t} \quad i=0,1
$$

so that we get the residual sum of squares

$$
S_{i j}=M_{i j}-M_{i k} M_{k k}^{-1} M_{k j}
$$

where $S_{i j}$ depends on $d$ and $b$. For fixed $d, b, \alpha$ and $\beta, \Gamma$ is defined as

$$
\Gamma(d, b, \alpha, \beta)=\left(M_{0 k}-\alpha \beta^{\prime} M_{1 k}\right) M_{k k}^{-1}
$$


For fixed $d, b$ and $\beta, \alpha$ is estimated as

$$
\hat{\alpha}(d, b, \beta)=S_{01} \beta\left(\beta^{\prime} S_{11} \beta\right)^{-1}
$$

and

$$
\hat{\Omega}(d, b, \beta)=S_{00}-\hat{\alpha}\left(\beta^{\prime} S_{11} \beta\right) \hat{\alpha}^{\prime}
$$

Plugging this estimates into the likelihood we get

$$
L(d, b, \hat{\alpha}, \beta, \hat{\Omega})=\left|S_{00}-S_{01} \beta\left(\beta^{\prime} S_{11} \beta\right)^{-1} \beta^{\prime} S_{10}\right|
$$

that is maximized by the eigenvector corresponding to the $r$ maximum eigenvalues that solve the problem

$$
\left|\lambda S_{11}-S_{10} S_{00}^{-1} S_{01}\right|=0
$$

The vector $\beta$ is estimated as the r-dimensional space spanned by the eigenvectors, $v_{j}$ for $j=$ $1, \ldots, r$, corresponding to the $r$ largest eigenvalues of $S_{11}^{-1} S_{10} S_{00}^{-1} S_{01}$.

Given this choice of $\beta$, the likelihood function is maximized only with respect to $d$ and $b$, that is

$$
(\hat{d}, \hat{b})=\arg _{d, b} \max L(d, b)
$$

where

$$
L_{T}(d, b)=\left[\left|S_{00}\right| \prod_{i=1}^{r}\left(1-\lambda_{i}\right)\right]^{-\frac{T}{2}}
$$

Once $d$ and $b$ are estimated, as the values that maximize the function $L(b, d)$, all the other parameter of model (7) are obtained as functions of $\hat{d}$ and $\hat{b}$.

Given the equality of integration orders, the presence of fractional cointegration in the FVECM is tested carrying out as a likelihood ratio test similar to that presented in Lasak (2009) for the case $d=1$. This test extends, to the fractional cointegration case, the well-known Johansen (1991) test for the cointegration rank. In particular, we consider the so called maximum eigenvalue 
statistic to test the hypothesis of reduced rank of the matrix $\Pi=\alpha \beta^{\prime}$

$$
\begin{aligned}
& H_{0}: \operatorname{rank}(\Pi)=r_{0}=0 \\
& H_{1}: \operatorname{rank}(\Pi)=r_{1}=1
\end{aligned}
$$

where $\operatorname{rank}(\Pi)=1$ implies that $\Pi$ can be decomposed into $\alpha \beta^{\prime}$. The maximum eigenvalue statistic, $\sup \lambda_{\max }$, is defined as

$$
\sup \lambda_{\max }=L R[0 \mid 1]=-T \ln \left[1-\lambda_{1}(\hat{d}, \hat{b})\right]
$$

where $\hat{d}$ and $\hat{b}$ are the parameter that maximize the profile likelihood in equation (49) under the hypothesis of rank 1. 


\section{References}

Andersen, T. G., Bollerslev, T., Diebold, F. X., and Ebens, H. (2001a). The distribution of stock return volatility. Journal of Financial Economics, 61:43-76.

Andersen, T. G., Bollerslev, T., Diebold, F. X., and Labys, P. (2001b). The distribution of exchange rate volatility. Journal of the American Statistical Association, 96:42-55.

Bai, J. and Perron, P. (2003). Computation and analysis of multiple structural change models. Journal of Applied Econometrics, 18(1):1-22.

Bandi, F. M. and Perron, B. (2006). Long memory and the relation between implied and realized volatility. Journal of Financial Econometrics, 4:636-670.

Barndorff-Nielsen, O. E. and Shephard, N. (2002). Estimating quadratic variation using realized variance. Journal of Applied Econometrics, 17(5):457-477.

Black, F. and Scholes, M. (1973). The pricing of options and corporate liabilities. Journal of Political Economy, 81:637654.

Bollerslev, T., Tauchen, G., and Zhou, H. (2009). Expected stock returns and variance risk premia. Review of Financial Studies, 22(11):4463-4492;.

Breitung, J. and Hassler, U. (2002). Inference on the cointegration rank in fractionally integrated processes. Journal of Econometrics, 110(2):167-185.

Busch, T., Christensen, B. J., and Nielsen, M. (2010). The role of implied volatility in forecasting future realized volatility and jumps in foreign exchange, stock, and bond markets. Journal of Econometrics, forthcoming.

Christensen, B. and Prabhala, N. (1998). The relation between implied and realized volatility. Journal of Financial Economics, 50:125150.

Christensen, B. J. and Nielsen, M. . (2006). Semiparametric analysis of stationary fractional cointegration and the implied-realized volatility relation. Journal of Econometrics.

Christensen, K. and Podolskij, M. (2007). Realized range based estimation of integrated variance. Journal of Econometrics, 141:323-349. 
Davidson, J., Peel, D., and Byers, J. (2006). Support for governments and leaders: Fractional cointegration analysis of poll evidence from the uk, 1960-2004. Studies in Nonlinear Dynamics E Econometrics, 10(1):1345-1345.

Dolado, J. J., Gonzalo, J., and Mayoral, L. (2005). What is what?: A simple time-domain test of long-memory vs. structural breaks. Economics Working Papers 954, Department of Economics and Business, Universitat Pompeu Fabra.

Duecker, M. and Startz, R. (1998). Maximum likelihood estimation of fractional cointegration with an application to us and canadian bond rates. Review of Economics and Statistics, 80:420426.

Engle, R. and Granger, C. (1987). Cointegration and error correction: representation estimation, and testing. Econometrica, 55:251276.

French, K., Schwert, G., and Stambaugh, R. (1987). Expected stock returns and volatility. Journal of Financial Economics, 19:3-30.

Gonzalo, J. and Lee, T.-H. (1998). Pitfalls in testing for long run relationships. Journal of Econometrics, 86(1):129-154.

Granger, C. W. and Hyung, N. (2004). Occasional structural breaks and long memory with application to the s\&p 500 absolute stock returns. Journal of Empirical Finance, 11:399-421.

Granger, C. W. J. (1981). Some properties of time series data and their use in econometric model specification. Journal of Econometrics, 16:121130.

Granger, C. W. J. (1986). Developments in the study of cointegrated economic variables. Oxford Bulletin of Economics and Statistics, 48(3):213-28.

Hamilton, J. (1989). A new approach to the economic analysis of nonstationary time series and the business-cycle. Econometrica, 57:357-384.

Hendry, D. F. and Massmann, M. (2007). Co-breaking: Recent advances and a synopsis of the literature. Journal of Business \& Economic Statistics, 25:33-51.

Johansen, S. (1988). Statistical analysis of cointegration vectors. Journal of Economic Dynamics and Control, 12:231-254. 
Johansen, S. (1991). Estimation and hypothesis testing of cointegration vectors in gaussian vector autoregressive models. Econometrica, 59(6):1551-80.

Johansen, S. (2008). A representation theory for a class of vector autoregressive models for fractional processes. Econometric Theory, Vol 24,3 .

Johansen, S. and Nielsen, M. r. (2010). Likelihood inference for a fractionally cointegrated vector autoregressive model. CREATES Research Papers 2010-24, School of Economics and Management, University of Aarhus.

Lasak, K. (2008). Maximum likelihood estimation of fractionally cointegrated systems. Technical report, CREATES Research Paper 2008-53.

Lasak, K. (2009). Likelihood based testing for no fractional cointegration. Technical report, CREATES Research Paper 2008-52, Forthcoming on Journal of Econometrics.

Lu, Y. K. and Perron, P. (2008). Modeling and forecasting stock return volatility using a random level shift model. Technical report, Department of Economics, Boston University.

Marinucci, D. and Robinson, P. M. (2001). Semiparametric fractional cointegration analysis. Journal of Econometrics, 105:225-247.

Merton, R. C. (1980). On estimating the expected return on the market. Journal of Financial Economics, 8:323-361.

Mikosch, T. and Starica, C. (2004). Nonstationarities in financial time series, the long-range dependence, and the igarch effects. The Review of Economics and Statistics, 86(1):378-390.

Morana, C. (2002). Common persistent factors in inflation and excess nominal money growth and a new measure of core inflation. Studies in Nonlinear Dynamics $\mathcal{E}$ Econometrics, 6:1-38.

Morana, C. (2007). A structural common factor approach to core inflation estimation and forecasting. Applied Economics Letters, 14:163-169.

Nielsen, M. . (2007). Local whittle analysis of stationary fractional cointegration and the impliedrealized volatility relation. Journal of Business 83 Economic Statistics, Vol. 25, No. $4: 427-446$.

Nielsen, M. . and Shimotsu, K. (2007). Determining the cointegration rank in nonstationary fractional system by the exact local whittle approach. Journal of Econometrics, 141:574-596. 
Ohanissian, A., Russell, J. R., and Tsay, R. S. (2008). True or spurious long memory? a new test. Journal of Business \& Economic Statistics, 26:161-175.

Parkinson, M. (1980). The extreme value method for estimating the variance of the rate of return. The Journal of Business, 53:61-65.

Perron, P. (1990). Testing for a unit root in a time series regression with a changing mean. Journal of Business and Economic Statistics, 8:153-162.

Perron, P. and Qu, Z. (2010). Long-memory and level shifts in the volatility of stock market return indices. Journal of Business \& Economic Statistics, 28(2):275-290.

Robinson, P. M. (1994). Rates of convergence and optimal spectral bandwidth for long range dependence. Probability Theory and Related Fields, 99:443-473.

Robinson, P. M. and Marinucci, D. (2003). Semiparametric frequency domain analysis of fractional cointegration. In Robinson, P. M., editor, Time Series with Long Memory, pages 334-373. Oxford University Press.

Robinson, P. M. and Yajima, Y. (2002). Determination of cointegrating rank in fractional systems. Journal of Econometrics, 106:217-241.

Rossi, E. and Santucci de Magistris, P. (2009). A no arbitrage fractional cointegration analysis of the range based volatility. Technical report, University of Pavia, Italy.

Schwert, G. (1989). Why does stock market volatility change over time? Journal of Finance, 44:1115-1153.

Shimotsu, K. (2006). Simple (but effective) tests of long memory versus structural breaks. Working Papers 1101, Queen's University, Department of Economics.

Shimotsu, K. and Phillips, P. (2005). Exact local whittle estimation of fractional integration. Annals of Statistics, 33:1890-1933. 


\begin{tabular}{lcccc}
\hline & Mean & Std. Dev. & Skewness & Exc.Kurtosis \\
\hline$I V_{t}$ & 0.1632 & 0.0573 & 1.1417 & 1.9716 \\
$R V_{t}$ & 0.1406 & 0.0644 & 1.6129 & 2.8787 \\
$\log I V_{t}$ & -1.8683 & 0.3314 & 0.2890 & -0.5454 \\
$\log R V_{t}$ & -2.0474 & 0.4011 & 0.5590 & -0.0990 \\
\hline$V I X_{t}$ & 0.1573 & 0.0534 & 0.9893 & 0.9652 \\
$R R V_{t}$ & 0.1261 & 0.0556 & 1.4617 & 2.3398 \\
$\log V I X_{t}$ & -1.9023 & 0.3218 & 0.3197 & -0.7598 \\
$\log R R V_{t}$ & -2.1524 & 0.3951 & 0.4772 & -0.2837 \\
\hline
\end{tabular}

Table 1: Sample statistics for the implied and realized volatilities.

Futures

\begin{tabular}{lccc}
\hline & $m_{d}=T^{0.7}=45$ & $m_{d}=T^{0.6}=26$ & $m_{d}=T^{0.5}=15$ \\
\hline$\hat{d}_{I V}$ & 0.7168 & 0.7576 & 0.7574 \\
& $(0.0745)$ & $(0.0980)$ & $(0.1265)$ \\
$\hat{d}_{R V}$ & 0.5616 & 0.5753 & 0.6931 \\
& $(0.0745)$ & $(0.0980)$ & $(0.1265)$ \\
$\widehat{T}_{0}$ & 3.1749 & 2.8628 & 0.5508 \\
& & Spot & \\
\hline & $m_{d}=T^{0.7}=41$ & $m_{d}=T^{0.6}=24$ & $m_{d}=T^{0.5}=14$ \\
\hline$\hat{d}_{V I X}$ & 0.6515 & 0.6515 & 0.9155 \\
& $(0.0781)$ & $(0.1002)$ & $(0.1336)$ \\
$\hat{d}_{R R V}$ & 0.5532 & 0.5750 & 0.7134 \\
$\widehat{T}_{0}$ & $(0.0781)$ & $(0.1002)$ & $(0.1336)$ \\
\hline & 0.9184 & 1.2419 & 2.1657 \\
\hline
\end{tabular}

Table 2: Fractional integration estimation with exact local Whittle estimator (standard error in parenthesis). The $\widehat{T}_{0}$ test statistic is calculated with $h(T)=\log (T)$. 


\begin{tabular}{ccc}
\hline & $\log R V_{t}$ & $\log R R V_{t}$ \\
\hline Split & 0.0924 & 0.1622 \\
d-diff & -1.9428 & -1.1013 \\
\hline & $\log I V_{t}$ & $\log V I X_{t}$ \\
\hline Split & 0.4077 & 0.8554 \\
d-diff & -1.2800 & -1.4064 \\
\hline
\end{tabular}

Table 3: Tests for truly vs spurious long memory. Split is the $p$-value of the Shimotsu (2006) based on sample splitting. $d$-diff is the value of the Shimotsu (2006) test based on $d$-differencing and the KPSS test. All tests are based on $m_{d}=T^{0.6}$ as a choice of the bandwidth for the estimation of the fractional integration order.

(a) $\log R V_{t}=\alpha+\beta \log I V_{t}+e_{t}$

\begin{tabular}{lccc}
\hline Bandwidth & $\hat{\alpha}_{m}$ & $\hat{\beta}_{m}$ & $\widehat{d}_{e}$ \\
\hline$m=20$ & -0.0478 & 1.0700 & $0.1897(0.0980)$ \\
& $(-0.3058,0.4329)$ & $(0.9284,1.3405)$ & \\
$m=15$ & -0.0530 & 1.0672 & $0.1889(0.0980)$ \\
& $(-0.3125,0.5127)$ & $(0.9067,1.3750)$ & \\
$m=9$ & -0.0125 & 1.0888 & $0.1951^{*}(0.0980)$ \\
& $(-0.4367,0.4551)$ & $(0.8285,1.3520)$ & \\
$m=6$ & -0.0046 & 1.0931 & $0.1964^{*}(0.0980)$ \\
& $(-0.4763,0.5075)$ & $(0.8411,1.4002)$ & \\
\hline
\end{tabular}

(b) $\log R R V_{t}=\alpha+\beta \log V I X_{t}+u_{t}$

\begin{tabular}{lccc}
\hline Bandwidth & $\hat{\alpha}_{m}$ & $\hat{\beta}_{m}$ & $\widehat{d}_{u}$ \\
\hline$m=20$ & -0.0632 & 1.0999 & $0.0161(0.1002)$ \\
& $(-0.4331,0.2261)$ & $(0.8923,1.2426)$ & \\
$m=15$ & -0.0136 & 1.1260 & $0.0582(0.1002)$ \\
& $(-0.4965,0.2605)$ & $(0.758,1.2630)$ & \\
$m=9$ & -0.0300 & 1.1173 & $0.0464(0.1002)$ \\
& $(-1.0051,0.4133)$ & $(0.4800 .3510)$ & \\
$m=6$ & -0.0196 & 1.1228 & $0.0541(0.1002)$ \\
& $(-1.2890,0.6742)$ & $(0.2784,1.5183)$ & \\
\hline
\end{tabular}

Table 4: Fractional Cointegration Analysis: Panel $(a)$ reports the results of the FDLS procedure in the case of future contracts, while Panel $(b)$ reports the results of the FDLS procedure in the case of spot contracts. In round brackets the $95 \%$ confidence intervals based on subsampling with size $\left[n^{\frac{2}{3}}\right]$ 

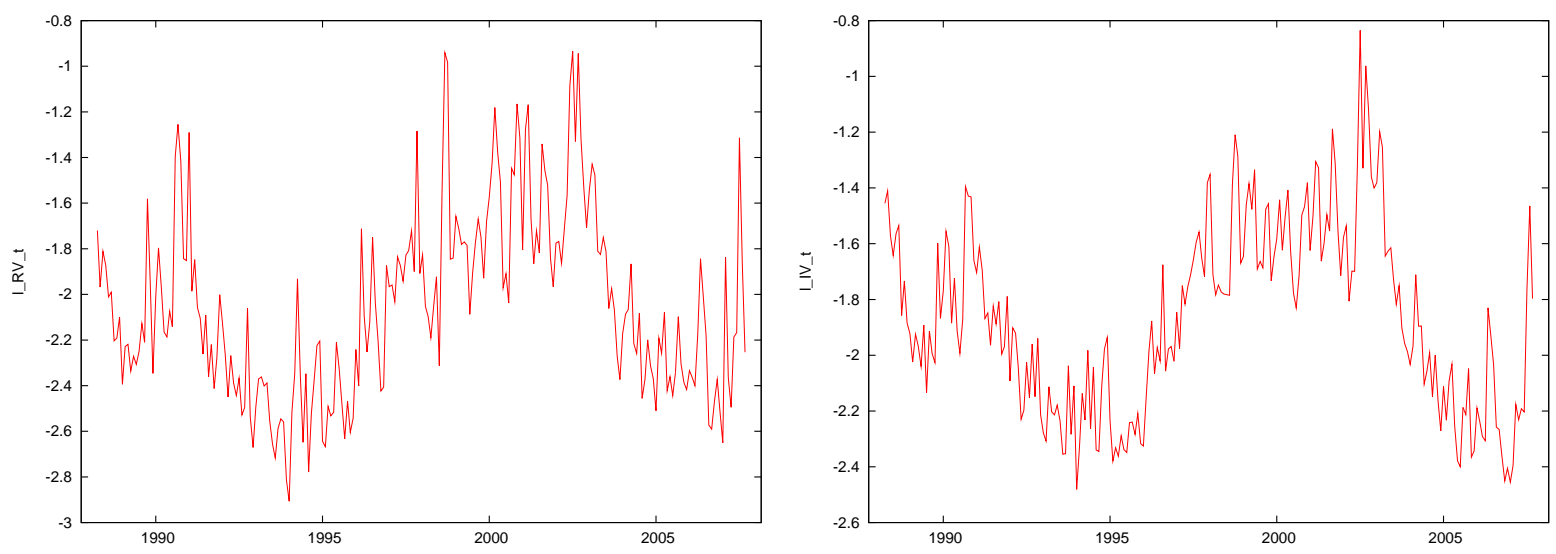

(a) Future log-volatilities
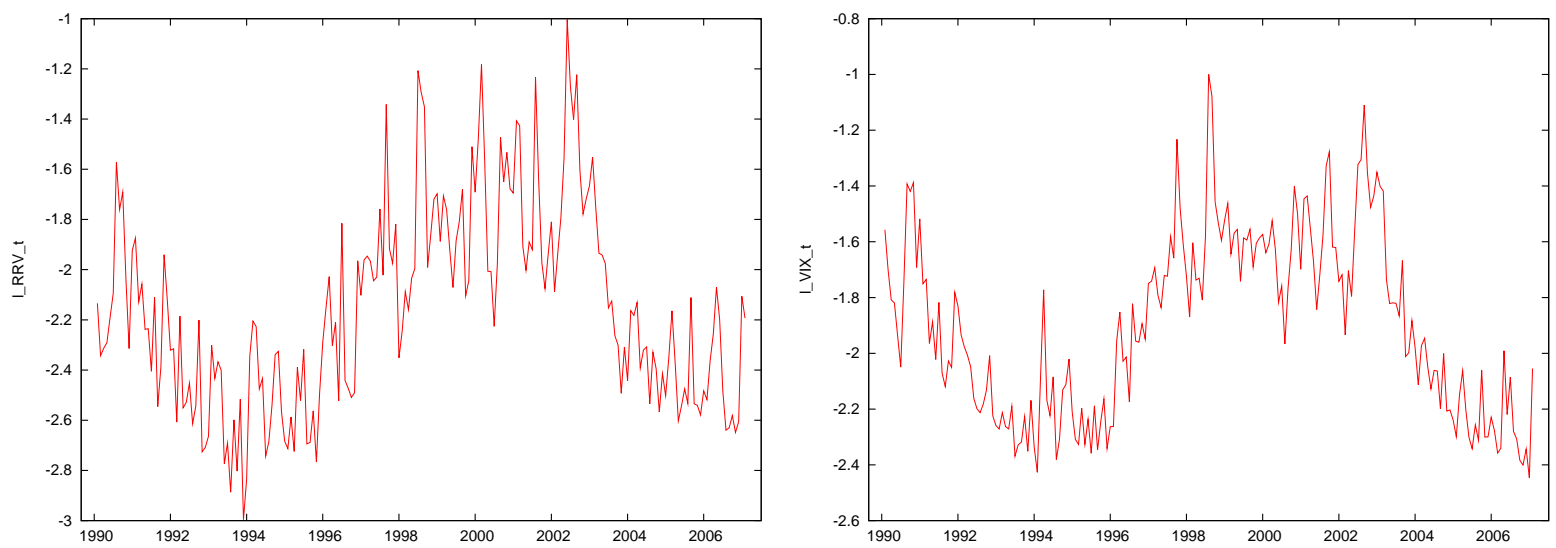

(b) Spot log-volatilities

Figure 1: Monthly realized-implied volatility measures: Panel (a) reports the series of log-volatility of the future contracts on S\&P from 4/1988 to 10/2007, Panel (b) reports the series of log-volatility of the spot contracts on S\&P from 1/1990 to 3/2007 

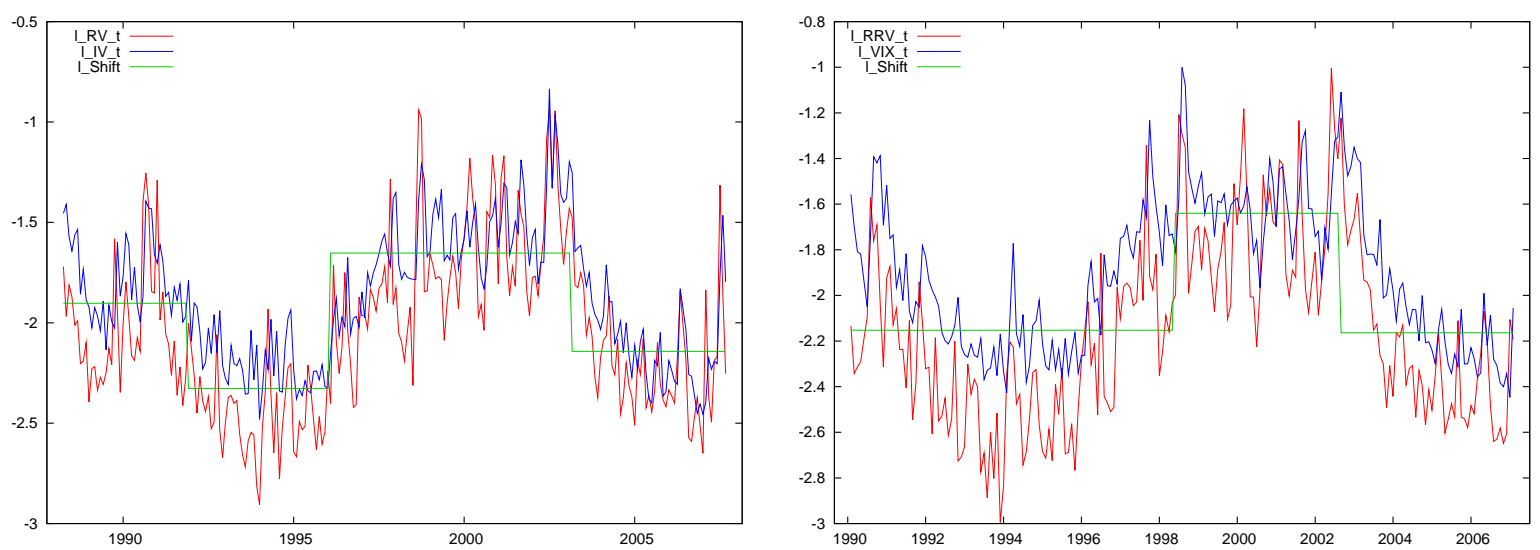

(a) Log Volatilities
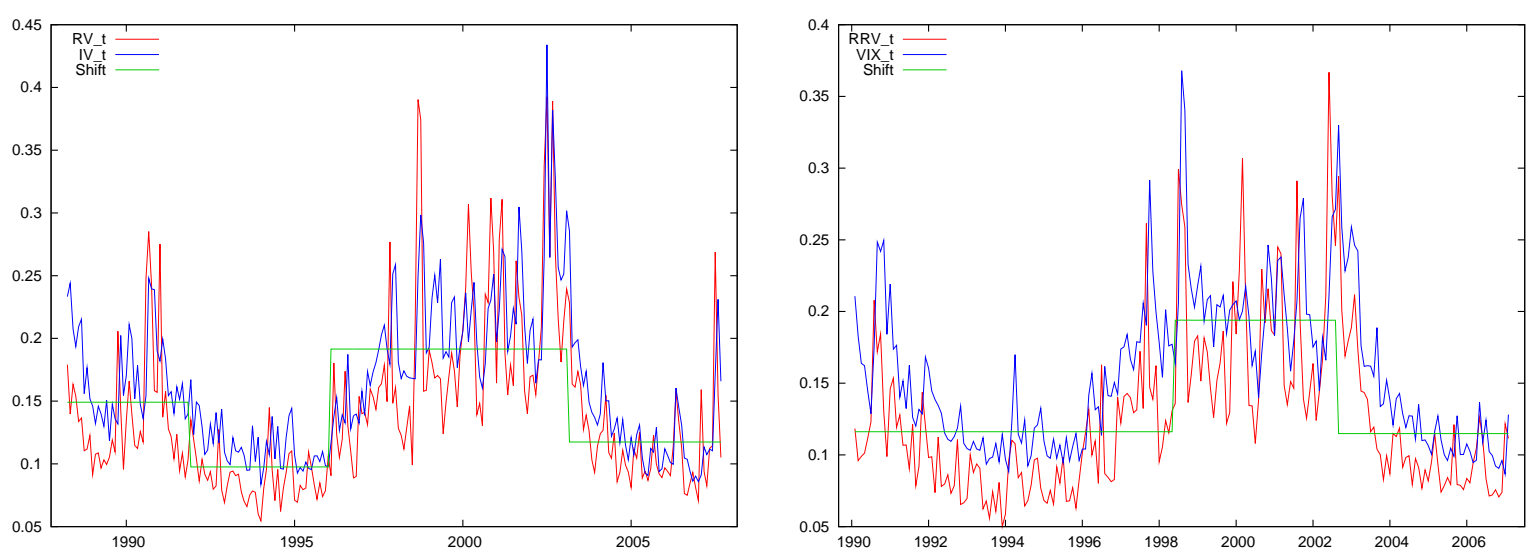

(b) Volatilities

Figure 2: Estimated shift processes: Panel (a) reports the identifies shifts on the log-volatilities, while panel (b) show the consequences of the selected break dates on the original volatility series. 


\begin{tabular}{lcccccc}
\hline \multicolumn{3}{c}{ FUTURES } & \multicolumn{3}{c}{ SPOT } \\
\hline$\hat{d}$ & Value & $Q_{5}$ & $Q_{95}$ & Value & $Q_{5}$ & $Q_{95}$ \\
$\hat{b}$ & 0.8047 & 0.7318 & 0.9354 & 0.8985 & 0.8147 & 1.0061 \\
$\hat{\beta}$ & -1.0227 & -1.1120 & -0.9479 & -0.9976 & -1.0663 & -0.8676 \\
$\hat{\alpha_{R}}$ & -0.2447 & -0.5694 & 0.0684 & -0.4108 & -0.8223 & -0.1641 \\
$\hat{\alpha_{I}}$ & 1.0384 & 0.6034 & 1.5554 & 0.8012 & 0.5303 & 1.4094 \\
$\hat{\mu}$ & 0.0803 & -0.0589 & 0.2205 & 0.2276 & 0.0043 & 0.5054 \\
\hline$\hat{\lambda}_{\text {max }}$ & 150.47 & - & 15.756 & 150.833 & - & 22.4168 \\
\hline
\end{tabular}

Table 5: Estimation Results of model 7 with intercept, $\mu$, in the cointegration relation. Table reports the estimated parameter value and the $5^{\text {th }}$ and $95^{\text {th }}$ bootstrapped quantiles, $Q_{5}$ and $Q_{95}$. Table reports also the value of the maximum eigenvalue statistic $\sup \lambda_{\max }$ for $r_{0}=0$ with the corresponding bootstrapped $95^{\text {th }}$ quantile under $H_{0}$ 
(a)

\begin{tabular}{lcccc}
\hline & \multicolumn{2}{c}{ FUTURES } & \multicolumn{2}{c}{ SPOT } \\
\hline & Value & $p$-val & Value & $p$-val \\
\hline$\hat{\nu}$ & 0.0340 & 0.0000 & 0.0447 & 0.0000 \\
$\hat{\sigma}_{\eta}$ & 0.5619 & 0.0165 & 0.4416 & 0.0006 \\
$\hat{\sigma}_{\epsilon}^{R V}$ & 0.2422 & 0.0000 & 0.2372 & 0.0000 \\
$\hat{\sigma}_{\epsilon}^{I V}$ & 0.1411 & 0.0000 & 0.1343 & 0.0000 \\
$\hat{\sigma}_{\epsilon}^{R V, I V}$ & 0.0970 & 0.0000 & 0.0595 & 0.0000 \\
$W^{\rho}$ & 2.7826 & 0.0476 & 2.7615 & 0.0483 \\
\hline
\end{tabular}

(b)

\begin{tabular}{lcccc}
\hline & \multicolumn{2}{c}{ FUTURES } & \multicolumn{2}{c}{ SPOT } \\
\hline & Value & $p$-val & Value & $p$-val \\
\hline$\hat{\nu}$ & 0.0111 & 0.0000 & 0.0089 & 0.0000 \\
$\hat{\sigma}_{\eta}$ & 0.3952 & 0.0632 & 0.3977 & 0.0979 \\
$\hat{\sigma}_{\epsilon}^{R V}$ & 0.2517 & 0.0000 & 0.2323 & 0.0000 \\
$\hat{\sigma}_{\epsilon}^{I V}$ & 0.1334 & 0.0000 & 0.1071 & 0.0000 \\
$\hat{\sigma}_{\epsilon}^{R V, I V}$ & 0.1293 & 0.0000 & 0.0760 & 0.0000 \\
$\phi_{11}$ & 0.6943 & 0.0000 & 0.6469 & 0.0000 \\
$\phi_{12}$ & 0.2150 & 0.0286 & 0.3369 & 0.0001 \\
$\phi_{21}$ & 0.4736 & 0.0000 & 0.4651 & 0.0000 \\
$\phi_{22}$ & 0.3708 & 0.0000 & 0.4351 & 0.0000 \\
$W^{\rho}$ & 0.0120 & 0.4565 & 0.2138 & 0.3219 \\
\hline
\end{tabular}

Table 6: Estimation Results: Bivariate Random Shift Model. Panel (a) reports the results of the common shift model without lags, while Panel $(b)$ presents the estimation results when we allow for VAR dynamics in the short term component. 
(a)

\begin{tabular}{lcccc}
\hline & \multicolumn{2}{c}{ FUTURES } & \multicolumn{2}{c}{ SPOT } \\
\hline & Value & $p$-val & Value & $p$-val \\
\hline$\gamma_{1}$ & -0.3730 & 0.0001 & -0.3113 & 0.0022 \\
$\phi_{11}$ & 0.6477 & 0.0000 & 0.6265 & 0.0000 \\
$\phi_{12}$ & 0.1873 & 0.0392 & 0.2597 & 0.0043 \\
$\gamma_{2}$ & -0.2044 & 0.0001 & -0.1141 & 0.0341 \\
$\phi_{21}$ & 0.4478 & 0.0000 & 0.4505 & 0.0000 \\
$\phi_{22}$ & 0.4009 & 0.0000 & 0.4305 & 0.0000 \\
\hline
\end{tabular}

(b)

\begin{tabular}{lcccc}
\hline \multicolumn{2}{c}{ FUTURES } & \multicolumn{2}{c}{ SPOT } \\
\hline & Value & $p$-val & Value & $p$-val \\
\hline$\gamma_{1}$ & -0.0418 & 0.0000 & -0.0765 & 0.0051 \\
$\phi_{11}$ & 0.5416 & 0.0000 & 0.5563 & 0.0000 \\
$\phi_{12}$ & 0.0004 & 0.9964 & 0.1577 & 0.0747 \\
$\gamma_{2}$ & 0.0904 & 0.0000 & 0.1214 & 0.0000 \\
$\phi_{21}$ & 0.3741 & 0.0000 & 0.4294 & 0.0000 \\
$\phi_{22}$ & 0.3537 & 0.0000 & 0.4540 & 0.0000 \\
\hline
\end{tabular}

Table 7: VAR dynamics. Panel (a) reports the estimated parameters of a VAR(1) on the original series, while Panel $(b)$ presents the estimation results of a VAR(1) on the detrended series.

\begin{tabular}{lcccc}
\hline & \multicolumn{2}{c}{ FUTURES } & \multicolumn{2}{c}{ SPOT } \\
& $t_{\left(d_{y}-d_{\epsilon}\right)}$ & $\lambda_{\max }>\lambda_{\max }^{*}$ & $t_{\left(d_{y}-d_{\epsilon}\right)}$ & $\lambda_{\max }>\lambda_{\max }^{*}$ \\
\hline FVECM $\left(\alpha_{1}, \alpha_{2}\right)=0$ & 6.4 & 5.0 & 4.4 & 4.3 \\
REGRESSION & 7.8 & 4.4 & 7.9 & 5.3 \\
LEVEL SHIFTS & 5.0 & 74.0 & 3.5 & 70.2 \\
LEVEL SHIFTS + VAR & 99.6 & 100 & 99.9 & 100 \\
VAR & 100 & 100 & 100 & 100 \\
\hline
\end{tabular}

Table 8: Percentage of rejections by regression and sup maximum eigenvalue tests under the null hypothesis of no cointegration and no common shifts. Nominal size $5 \%$ 


\begin{tabular}{lcccc}
\hline & \multicolumn{2}{c}{ FUTURES } & \multicolumn{2}{c}{ SPOT } \\
& $t_{\left(d_{y}-d_{\epsilon}\right)}$ & $\lambda_{\max }>\lambda_{\max }^{*}$ & $t_{\left(d_{y}-d_{\epsilon}\right)}$ & $\lambda_{\max }>\lambda_{\max }^{*}$ \\
\hline FVECM $\left(\alpha_{1}, \alpha_{2}\right)=0$ & 96.6 & 100 & 95.8 & 100 \\
LEVEL SHIFTS & 98.8 & 99.3 & 99.6 & 99.9 \\
LEVEL SHIFTS + VAR & 99.7 & 100 & 100 & 100 \\
VAR & 99 & 100 & 100 & 100 \\
\hline
\end{tabular}

Table 9: Percentage of rejections by regression and sup maximum eigenvalue tests of the null under fractional cointegration 


\begin{tabular}{lccccc}
\hline & $t_{\left(d_{y}-d_{\epsilon}\right)}$ & $\lambda_{\max }>\lambda_{\max }^{*}$ & & $t_{\left(d_{y}-d_{\epsilon}\right)}$ & $\lambda_{\max }>\lambda_{\max }^{*}$ \\
$\alpha / 2$ & 85.5 & 99.6 & $\sigma_{\eta} / 2$ & 85.0 & 99.5 \\
$\alpha / 4$ & 49.5 & 86.1 & $\sigma_{\eta} / 4$ & 50.6 & 77.5 \\
$\alpha / 6$ & 35.7 & 53.9 & $\sigma_{\eta} / 6$ & 30.6 & 44.7 \\
$\alpha / 8$ & 25.8 & 31.3 & $\sigma_{\eta} / 8$ & 22.8 & 29.0 \\
$\alpha / 10$ & 21.3 & 22.7 & $\sigma_{\eta} / 10$ & 18.4 & 19.0 \\
\hline
\end{tabular}

Table 10: Percentage of rejections by regression and sup maximum eigenvalue tests of the null under fractional cointegration for different values of $\alpha$ and $\sigma_{\eta}$ 
2010-46: Christian Bach and Stig Vinther Møller: Habit-based Asset Pricing with Limited Participation Consumption

2010-47: Christian M. Dahl, Hans Christian Kongsted and Anders Sørensen: ICT and Productivity Growth in the 1990's: Panel Data Evidence on Europe

2010-48: Christian M. Dahl and Emma M. Iglesias: Asymptotic normality of the QMLE in the level-effect ARCH model

2010-49: $\quad$ Christian D. Dick, Maik Schmeling and Andreas Schrimpf: Macro Expectations, Aggregate Uncertainty, and Expected Term Premia

2010-50: Bent J esper Christensen and Petra Posedel: The Risk-Return Tradeoff and Leverage Effect in a Stochastic Volatility-in-Mean Model

2010-51: $\quad$ Christos Ntantamis: A Duration Hidden Markov Model for the Identification of Regimes in Stock Market Returns

2010-52: Christos Ntantamis: Detecting Structural Breaks using Hidden Markov Models

2010-53: Christos Ntantamis: Detecting Housing Submarkets using Unsupervised Learning of Finite Mixture Models

2010-54: $\quad$ Stefan Holst Bache: Minimax Regression Quantiles

2010-55: Nektarios Aslanidis and Charlotte Christiansen: Sign and Quantiles of the Realized Stock-Bond Correlation

2010-56: $\quad$ Anders Bredahl Kock: Oracle Efficient Variable Selection in Random and Fixed Effects Panel Data Models

2010-57: Charlotte Christiansen, J uanna Schröter J oensen and J esper Rangvid: The Effects of Marriage and Divorce on Financial Investments: Learning to Love or Hate Risk?

2010-58: Charlotte Christiansen, Maik Schmeling and Andreas Schrimpf: A Comprehensive Look at Financial Volatility Prediction by Economic Variables

2010-59: J ames G. MacKinnon and Morten Ørregaard Nielsen: Numerical distribution functions of fractional unit root and cointegration tests

2010-60: Bent J esper Christensen and Paolo Santucci de Magistris: Level Shifts in Volatility and the Implied-Realized Volatility Relation 